\title{
Thermal Improvement of InP Wire Photonic Crystal Laser on Silicon by addition of Diamond Nanoparticles in Polymer Bonding Layer
}

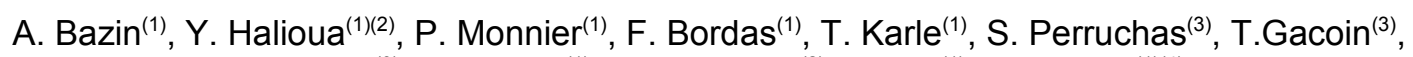 \\ H. Girard ${ }^{(3)}$, I. Sagnes ${ }^{(1)}$, G. Roelkens ${ }^{(2)}$, R. Raj ${ }^{(1)}$, F. Raineri(1)(4) \\ (1) Laboratoire de Photonique et de Nanostructures (CNRS UPR20), Route de Nozay, 91460, \\ Marcoussis, France, fabrice.raineri@lpn.cnrs.fr \\ (2) Photonics Research Group, Department of Information Technology, Ghent University, B-9000 \\ Ghent, Belgium \\ (3) Groupe de Chimie du Solide, Laboratoire de Physique de la Matière Condensée (UMR CNRS \\ 7643), Ecole Polytechnique, 91128 Palaiseau, France \\ (4) Université Paris-Diderot, Paris, France
}

\begin{abstract}
Diamond Nanoparticles are added to BCB polymer in order to increase the thermal dissipation of InP-based photonic crystal cavity laser bonded on silicon. Optical measurement are performed to evaluate the enhancement of the heat sinking with nanoparticles density.
\end{abstract}

Silicon photonics is a major subject of research in optoelectronics because it opens the way to the low cost fabrication of ultracompact optical integrated circuits. Integration of III-V materials on Silicon constitutes an important step on that road, through their direct band gap allowing light emission, and thus integration of active functions on passive Silicon devices ${ }^{1,2}$. One way to achieve it consists in the bonding of the III-V layer onto a Silicon On Insulator (SOI) substrate. The bonding technique of our choice uses planarising polymer benzocyclobutene (BCB) which is transparent at $1.5 \mu \mathrm{m}$. Inconveniently, BCB has low thermal conductivity, inducing poor heat dissipation (see Table 1) from the III-V layer. Indeed, overheating prevents continuouswave or ultra high bit-rate operation. Furthermore, it induces spectral shift and output power lowering which may be prejudicial in a photonic integrated circuit, particularly in a wavelength sensitive function, like WDM-based devices.

Tab. 1: A typical table caption

\begin{tabular}{|c|c|}
\hline Material & $\begin{array}{c}\text { Thermal } \\
\text { conductivity } \\
\text { W. } \mathrm{m}^{-1} \cdot \mathrm{K}^{-1}\end{array}$ \\
\hline $\mathrm{Si}$ & 130 \\
\hline $\mathrm{InP}$ & 63 \\
\hline $\mathrm{BCB}$ & 0,29 \\
\hline Diamond & $>1000$ \\
\hline
\end{tabular}

In this work, we study the possibility of including Diamond Nanoparticles (DN) in BCB bonding layer in order to increase its thermal dissipation without impacting optical losses. Indeed, bulk diamond exhibits one of the highest known thermal conductivity (> $\left.1000 \mathrm{~W} \cdot \mathrm{m}^{-1} \cdot \mathrm{K}^{-1}\right)$. The thermal study relies on the analyse of the spectral behaviour of the InP-based photonic crystal wire cavity laser emission with temperature and pump power.

Firstly, in order to measure the impact of the DN on the optical losses, we perform loss measurement on $450 \mathrm{~nm} \times 220 \mathrm{~nm}$ SOI waveguides with lengths ranging from $1 \mathrm{~mm}$ to 7 $\mathrm{mm}$ covered with different BCB/DN solutions where the concentration of the DN is varied from 0 to $10 \%$. We find losses of $16 \mathrm{~dB} / \mathrm{cm}$ for four concentrations, as deduced from Figure 1. This means that, for those concentrations, DN do not create additional losses. This was expected as, on the one hand, Diamond is transparent in the near infrared and, on the other hand, particles size is small enough to prevent scattering of light. 


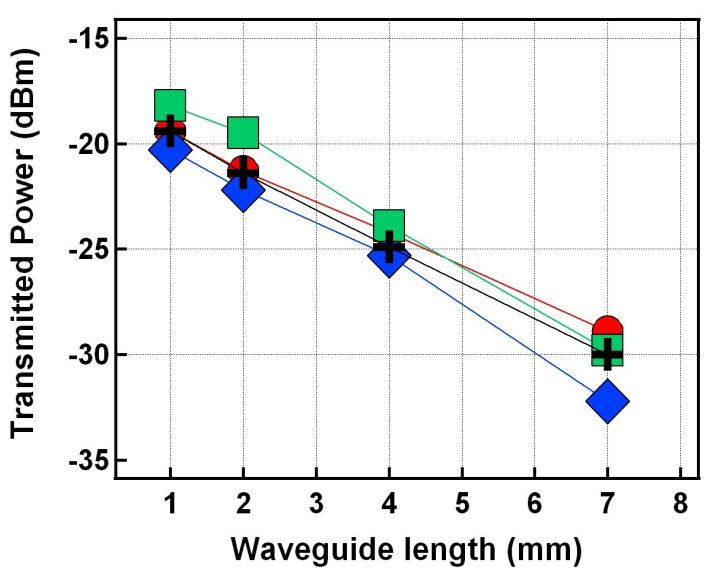

Fig. 2: Transmitted power (in $\mathrm{dBm}$ ) versus waveguide length for different DN concentration: $0 \%$ (circles), $2.5 \%$ (cross), $5 \%$ (diamond shape), $10 \%$ (square)

The thermal experiments consist in measuring the emission peak of a laser while varying pump power and substrate temperature.

We fabricate the lasers in a $255 \mathrm{~nm}$ high $\mathrm{InP}$ membrane embedding 4 quantum wells on bulk Silicon with BCB incorporating different densities of ND. We use electronic lithography to define the pattern and Reactive lon Etching to transfer it into the $\mathrm{SiN}_{\mathrm{x}}$ hard mask. We finally etch the III-V membrane using Inductively Coupled Plasma. The whole detailed process is described elsewhere ${ }^{3}$. The samples studied in this work are $\mathrm{InP} / \mathrm{lnGaAs} / \mathrm{lnGaAsP}$ nanowire photonic crystal lasers ${ }^{4}$ (figure 2) emitting around $1.5 \mu \mathrm{m}$. The cavity is formed by two sets of holes drilled in a $500 \mathrm{~nm}$ wide wire, each of them forming a high reflectivity mirror.

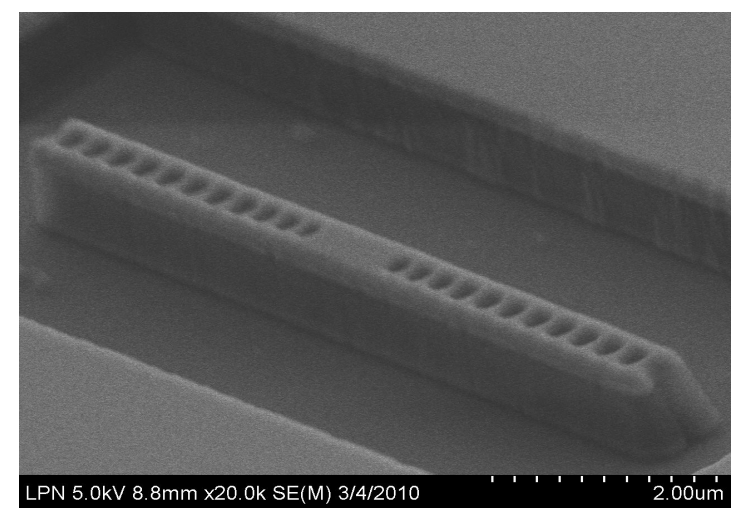

Fig. 2: SEM picture of the sample

We optically pump the cavity with a $800 \mathrm{~nm}$ laser diode which can be modulated to produce 30 ns pulses at repetition rates ranging from tens to several hundreds of $\mathrm{kHz}$. The pump beam is focused at normal incidence on the sample surface with a 10X infrared microscope objective. Emitted light is collected through the same objective and analysed by a spectrometer. The sample is placed on a copper plaque with a Peltier element for temperature control.

In order to calibrate wavelength shifting with temperature, we pump the cavity above laser threshold, at low repetition-rate of $33 \mathrm{kHz}$, making the induced local heating negligible. Figure 3 shows the emission peak wavelength as a function of the substrate temperature. We observe a red-shift with increasing temperature, due to the thermal expansion and change in refractive index of the material.

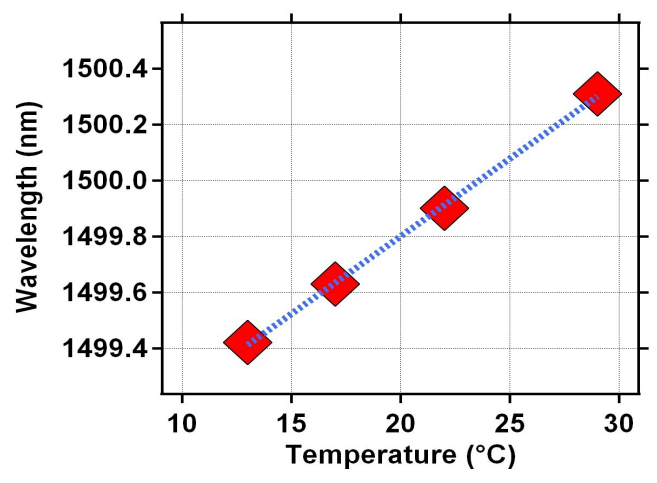

Fig. 3: Wavelength versus temperature of the sample in low duty-cycle regime.

Secondly, at fixed substrate temperature of $283 \mathrm{~K}$, we increase the duty cycle up to $7.5 \%$ by increasing the repetition rate, in order to observe local heating of the PC cavity. The increase in peak pump power induces a shift in the emission wavelength, which we relate to a change in temperature, thanks to the previous calibration measurement. The evolution of the average local change in temperature with the average pump power is described in figure 4 for three different $D N$ concentrations. We observe a linear increase in local temperature with pump power above a thermal threshold. As DN concentration increase, thermal threshold increase and the slope decrease, demonstrating a better thermal dissipation in BCB/DN polymer. 


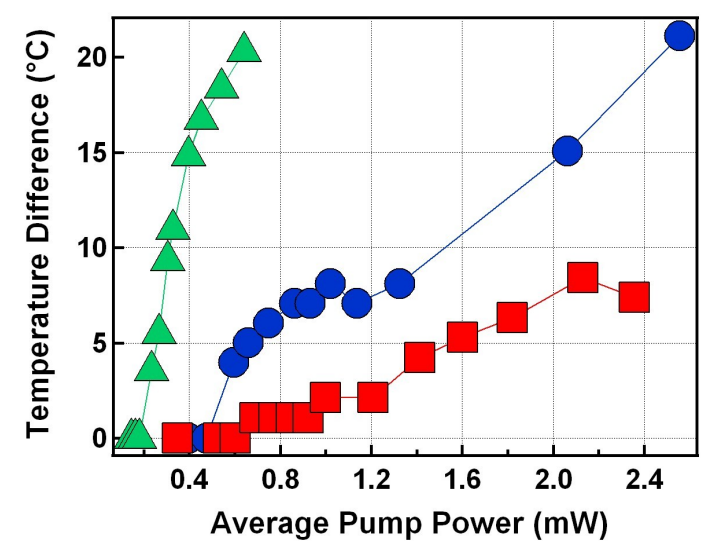

Fig. 3: Temperature difference versus average pump power at $7,5 \%$ duty cycle (30ns pulse) for $0 \%$ (triangle), $5 \%$ (circle), $10 \%$ (square)

Through the use of DN, we succeed in pumping our laser at higher average power such as 2,4 $\mathrm{mW}$ without inducing irreversible damage neither thermal degradation of the performance.
As a summary, we have demonstrated an improvement in thermal management of hybrid $\mathrm{III}-\mathrm{V} / \mathrm{Si}$ structures by addition of Diamond Nanoparticles in the BCB bonding layer. We think this is a promising solution for integration of quantum well photonic crystal continuouswave operating laser at room temperature in optical integrated circuits.

We acknowledge ICT FP7 HISTORIC european project and ANR Jeunes chercheurs PROWOC project for funding. We thank $A$. Beveratos for useful discussion.

\section{References}

1 A.W. Fang et al, Mater. Today, 10, 28 (2007).

2 Y. Halioua et al, Appl. Phys. Lett., 95, 201119 (2009).

3 K.-H. Lee, S. Guilet, G. Patriarche, I. Sagnes, and A. Talneau, J. Vac. Sci. Technol. B 26, 1326 (2008).

4 Y. Halioua et al, to be published (2010). 